\section{Antimalariamittel bei CLE erste Wahl}

$\mathrm{D}$ ie Behandlung des kutanen Lupus erythematodes (CLE) ist eine Herausforderung. Es gibt keine Therapie, die speziell für diese Indikation zugelassen ist, topische oder systemische Medikamente werden daher „off label“ eingesetzt.

In einer aktuellen S2k-Leitlinie wurden unter Federführung des European Dermatology Forum (EDF) in Zusammenarbeit mit der European Academy of Dermatology and Venereology (EADV) Expertenempfehlungen für die Behandlung des CLE entwickelt [Kuhn A et al. J Eur Acad Dermatol Venereol 2017; 31: 389-404]: Bei umschriebenen Hautveränderungen bleiben topische Kortikosteroide die wesentliche Säule der Therapie, alternativ können Calcineurinhibitoren eingesetzt werden. Für Patienten mit schweren und/oder ausgedehnten Hautläsionen, insbesondere bei hohem Risiko für eine Narbenbildung und/oder für die Entwicklung eines systemischen Lupus erythematodes, und für die Langzeittherapie werden als erste Wahl Antimalariamittel empfohlen. Bei hoch aktiven Formen können eventuell kurzzeitig systemische Kortikosteroide kombiniert werden. Als Zweit- und Drittlinienmedikamente für die systemische Therapie werden Methotrexat,
Retinoide, Dapson, Mycophenolatmofetil und Mycophenolsäure genannt. Thalidomid ist nur in Einzelfällen vertretbar.

Gerade bei rasch progredienten Verläufen sei eine frühzeitige und effektive systemische Therapie wichtig, betonte Prof. Michael Sticherling, Erlangen. Zu bevorzugen sei das Antimalariamittel Hydroxychloroquin, das besser verträglich sei als Chloroquin. Nachdem Hydroxychloroquin bereits seit langem beim CLE eingesetzt wird, wurde die Effektivität und Verträglichkeit jetzt endlich in einer doppelblinden, randomisierten Placebo-kontrollierten Studie aus Japan überprüft, berichtete Sticherling. In die Untersuchung [Yokogawa N et al. Arthritis Rheumatol 2017; 69: 79199] eingeschlossen waren 103 Patienten mit aktivem CLE und einem CLASI (Cutaneous Lupus Erythematosus Disease Area and Severity Index) von mindestens 4. Sie erhielten zunächst randomisiert im Verhältnis 3:1 über 16 Wochen Hydroxychloroquin oder Placebo. Anschließend wurden alle Patienten für 36 Wochen mit Hydroxychloroquin behandelt. Die Sicherheit wurde bis Woche 55 erfasst.

Der CLASI, der als primärer Endpunkt definiert wurde, hatte sich bis
Woche 16 in beiden Gruppen signifikant gegenüber dem Ausgangswert gebessert $(-4,6$ vs. $-3,3)$. Im IGA (Investigator's Global Assessment) zeigte sich ein gröBerer Anteil gebesserter oder deutlich gebesserter Patienten in der Hydroxychloroquin-Gruppe (51,4 vs. 8,7\%). Die Ergebnisse der weiteren sekundären Endpunkte - quantifizierende Fotoevaluation, PGA (Patient's Global Assessment) und Skindex-29-Score - sprachen ebenfalls für die Effektivität von Hydroxychloroquin. Zellulitis, Arzneimittelexantheme, Leberfunktionsstörungen und Steven-Johnson-Syndrom waren in seltenen Fällen schwere Unverträglichkeitsreaktionen unter der Hydroxychloroquin-Therapie.

Die Therapie mit Hydroxychloroquin sollte über mindestens drei Monate erfolgen, empfahl Sticherling. Die Dosierung sollte 6-6,5 mg pro kg Idealgewicht betragen. Bei Therapieversagen kann eine Kombination mit Quinacrin erwogen werden.

Inwieweit therapeutische Antikörper gegen Pathogenese-relevante Zytokine und Adhäsionsmoleküle künftig bedeutsam werden, bleibe abzuwarten, so Sticherling. Erste Phase-II-Studien dazu laufen bereits. Angelika Bauer-Delto

Vortrag „Autoimmundermatosen“, Michael Sticherling (Erlangen), Wiesbaden, 11.11.2017

\title{
Schützt Atopie vor Basalzellkarzinomen?
}

$\mathrm{D}$ iskutiert wird immer wieder der Zusammenhang zwischen atopischer Diathese und Hautkrebsrisiko. Die Befunde hierzu seien inkonsistent, so Prof. Günther Hofbauer, Zürich. Theoretisch sind zwei konträre Konzepte denkbar: Einerseits könnten Allergene Immunantworten stimulieren und so potenziell einen protektiven Effekt gegen Krebs durch eine erhöhte Abwehrfunktion haben. Andererseits könnte eine chronische Immunstimulation bei Atopie zu proonkogenen Mutationen führen, wie dies zum Beispiel bei entzündlichen Darmerkrankungen mit bekannt erhöhtem Kolonkarzinomrisiko vermutet wird.
In einer deutschen Querschnittstudie wurden nun die Daten von $90.265 \mathrm{Be}$ schäftigten ausgewertet, bei denen Ganzkörper-Hautkrebsscreenings durch Dermatologen durchgeführt worden waren und Angaben zur persönlichen Anamnese vorlagen. Bei 30,7\% waren in der Vorgeschichte Erkrankung aus dem atopischen Formenkreis diagnostiziert worden. Es zeigte sich, dass bei Atopikern die Prävalenz von aktinischen Keratosen, Basalzellkarzinomen und malignen Melanomen niedriger lag. Unter Berücksichtigung von Alter, Geschlecht und bekannten Risikofaktoren wie Hauttyp und Sonnen- brände in der Kindheit verblieb Atopie als signifikanter protektiver Faktor beim Basalzellkarzinom (Odds Ratio 0,77 ) sowie beim Melanom (Odds Ratio 0,53 ] [Schäfer et al. J Eur Acad Dermatol Venereol. 2017; doi: 10.1111/ jdv.14456].

Damit scheint klar belegt, dass Atopie einen Schutz vor Basalzellkarzinomen und auch vor Melanomen vermittelt, so Hofbauer. Ob hierfür die atopische Diathese an sich oder damit verknüpfte Faktoren wie Verhalten, Behandlung und Arztkonsultationen relevant sind, werden prospektive Studien klären müssen.

Angelika Bauer-Delto

Vortrag „Non-Melanoma Skin Cancer", Günther Hofbauer (Zürich), Wiesbaden, 11.11.2017 